\title{
Applying virtual fixtures to the distal end of a minimally invasive surgery instrument
}

\author{
Marie-Aude Vitrani, Cécile Poquet, Guillaume Morel
}

\begin{abstract}
The comanipulation paradigm, in which a user and a robot simultaneously hold a tool, allows for gesture guidance. In particular, virtual fixtures, which are geometrical constraints imposed to the tool by the robot, have received a great interest in the domain of surgical applications. So far, this concept has been implemented in the context of open surgery. This paper explores the application of virtual fixtures for minimally invasive surgery, where the tool is inserted in the patient through a fulcrum. Here, a key issue is to return to the surgeon forces that are virtually applied at the instrument distal tip, while the robot is physically attached to the instrument proximal handle.

To this aim, two approaches are investigated. A first approach consists in applying a full wrench at the proximal end of the instrument that is equal to the wrench constituted by a pure force applied to the instrument's distal tip. A second approach consists in applying a pure force to the instrument proximal end, thanks to a lever model about the fulcrum.

The two approaches are compared through experiments during which naive subjects blindly perform virtual object palpation and robot guided movements. During experiments, indicators involving motion and force analysis are computed. The user capacity to distinguish between several virtual objects is evaluated as well. Although drastically different, the two approaches provide assistance with a similar level of efficiency.
\end{abstract}

\section{INTRODUCTION}

\section{A. Virtual Fixtures and Comanipulation}

Virtual fixtures are geometrical constraints actively imposed by a robot to its end-effector [1]. They have been conceived in the context of telemanipulation, where they were applied to a motorized master arm. In this case, they constitute an additional haptic feedback provided to the user, thus easing the slave telemanipulator control [2]. In the present paper, virtual fixtures are considered in the context of comanipulation.

Comanipulation is a paradigm in which a robot and a subject simultaneously hold a tool and perform a task. The two most basic functions that a comanipulator can exhibit are a free mode, where the tool movements are not constrained at all, and the locked mode, where the robot prevents the tool from any movements [3]. In between, the robot can apply a partial constraint. A first approach consists in imposing a given geometrical equality constrain to the tool [4]. For example the robot can impose that a given point $T$ of the tool remains still, while the tool orientation around $T$ is freely

Authors are with (1) Sorbonne Universités UPMC Univ. Paris 06, UMR 7222, ISIR, Paris, France, (2) INSERM, U1150, Agathe-ISIR, F-75005, Paris France, (3) CNRS, UMR 7222, ISIR, F-75005, Paris, France. Email: vitrani@isir.upme.fr.

This work was partly supported by the project PROSBOT funded by ANR, TECSAN 2011 program, under reference ANR-11-TECS-0017 and by french state funds managed by the ANR within the Investissements d'Avenir programme (Labex CAMI) under reference ANR-11-LABX-0004.

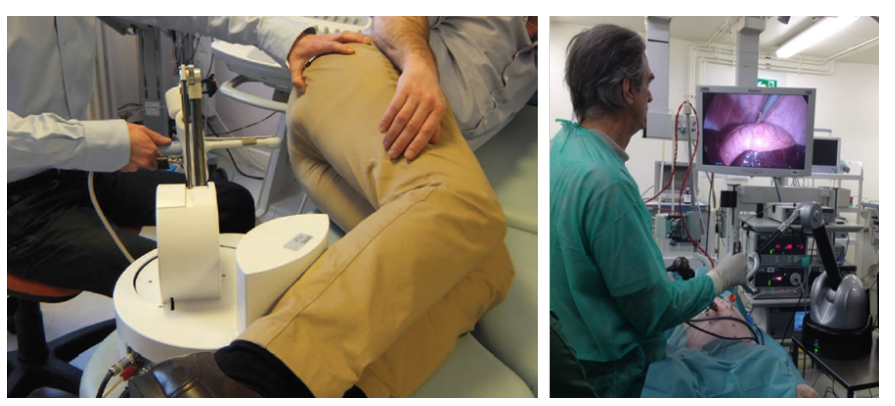

Figure 1. Comanipulating an instrument through a fulcrum: left - the 6-DOFs robot Apollo assists a urologist who manipulates an endorectal ultrasound probe to perform prostate biopsies [9]; right - the same concept applied to endoscopic surgery

set by the user. In a second approach, virtual fixtures can be conceived as inequality constraints, thus separating the space into free regions and forbidden regions. The reader interested in more details on these approaches and their implementation can consult a recent review on virtual fixtures in [5].

\section{B. Application to assistance to surgery}

The concepts of comanipulation and virtual fixtures have received an increasing interest in the particular context of surgical robotics [6]. Pioneer examples include PADyC, proposed in 2001 for cardiac surgery applications [7] and the steady hand robot aimed at assisting eye surgery [8].

Meanwhile, in the Operating Rooms, the comanipulation concept was successfully applied to orthopedic surgery, with a particular focus on assistance to bone milling, following the work on Acrobot and active constraints [10]-[12]. For this application, virtual fixtures are used to delimit forbidden regions, where the milling instrument shall not penetrate, in order to preserve bony tissue. As a result, the surgeon is assisted in sculpting a region whose geometry has been defined during a planning procedure, with more safety and more precision. In this scenario, a preoperative procedure is to be performed at the beginning of the operation to register the bone and the robot. Comanipulation and virtual fixtures for bone surgery are now available for clinical practice, e.g. with the Makoplasty system whose efficiency in increasing the gesture precision has been proven though clinical cases [13]. The gesture precision is even increased when a visual feedback is used in combination with a force feedback [14].

\section{Keyhole surgery: the fulcrum}

In this paper we focus on applying comanipulation and virtual fixtures in the context of so-called keyhole surgery, 
as illustrated in Fig. 1. Elongated instruments are introduced into the patient's body through natural orifices or small incisions. The instrument possesses only 4 Degrees of Freedom (DOFs): three independent rotations around the insertion point and one translation along the instrument longitudinal axis. A kinematic constraint is thus formed and challenges the surgeons' sensorimotor system. Combined with the lack of depth perception due to the indirect visual 2D feedback, this degrades the manipulation skills increases the duration of the learning process [15].

In order to assist surgeons' manipulation through a fulcrum, a number of robotic devices have been developed, with different kinematic designs. In some cases, such as [16], a 4 DOF robot exhibiting a remote center of motion (RCM) is used. This implies the robot base body to be carefully placed in the workspace prior to instrument manipulation, in such a way that its RCM coincides with patient's entry point. In other cases, a conventional 6-DOFs robot is used. This allows placing the robot base independently from the insertion point location. Such a 6-DOFs robot can be fully actuated, as in [17]. In this case, one has to solve for the kinematic constraint in real time, using either a knowledge on the fulcrum location, as proposed in [18]-[22], or an additional sensor to estimate this location, as proposed in [23]. The robot can also be partially actuated, as in [24]. Here, a first combination of 3 active DOFs is used to position the wrist center; 2 passive DOFs in the wrist allows free orientation of the instrument axis while the last DOF, corresponding to the instrument rotation around its axis, is motorized. Such a combination allows to respecting the kinematic constraint independently from the location of the fulcrum with respect to the robot base, while using four actuators only.

\section{Force control for manipulation involving a fulcrum}

Little literature is available on force control through a fulcrum. Most of it proposes to integrate a force sensor at the distal end of the instrument and to implement a distal force closed loop controller (see e.g. [18]). This question is treated independently from the robot kinematics and fulcrum constraint. The aim here is, in a context of force feedback teleoperated systems, to control the instrument-organ interaction despite disturbance forces applied at the fulcrum.

In the context of comanipulation, the question of force control is not limited to the distal interaction. Rather, it is required to deal with simultaneous distal (instrument-organs) and proximal (instrument-surgeon-robot) interactions, while minimizing the forces applied to the fulcrum. This question is treated in [25], where a 4 DOFs comanipulation robot exhibiting a RCM is presented for the implementation of a force feedback loop, when forces are applied both at the distal and proximal ends of an endoscopic surgery instrument. This configuration is shown to raise specific kinematic stability problems, formally studied in [26].

Meanwhile, to our knowledge, there is no literature dealing with the control of the wrench applied by a 6-DOFs robot to the handle of an instrument in order to produce virtual

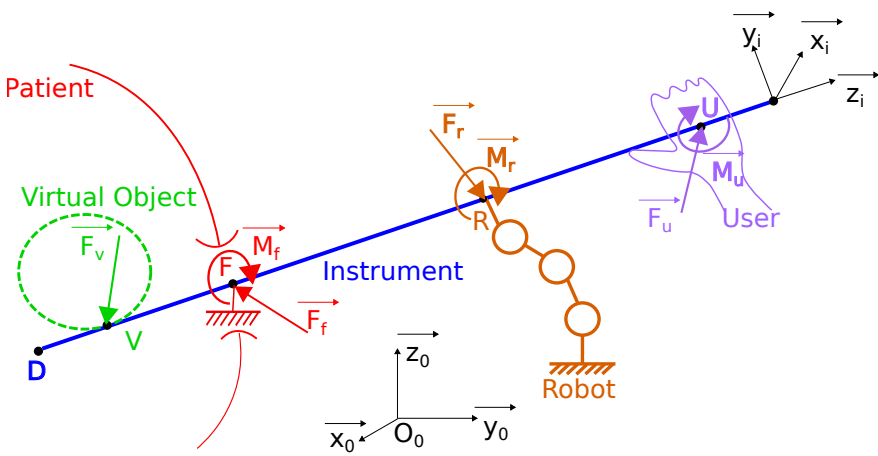

Figure 2. Wrenches applied to a comanipulated key-hole surgery instrument. Point $D$ denotes the distal end (physical tip) of the instrument inserted into the patient through the fulcrum point $F$. The user and the robot hold the proximal part (handle) of the instrument at point $U$ and point $R$, respectively. Point $V$ is where a force $\vec{F}_{v}$ is to be emulated in order to simulate the interaction with a virtual object

fixture to its distal end, the instrument being comanipulated through a fulcrum. Such a configuration is illustrated in Fig. 1, for two different applications: prostate biopsies, where the comanipulted instrument is an endorectal utrasound probe insterted through the anus and endoscopic surgery, where the robot and the surgeon comanipulate an elongated instrument through a trocar. In these applications, the use of a 6-DOFs robot rather than an RCM allows avoiding to precisely register the robot base with respect to the patient anatomy thus easing the installation. However, it has the disadvantage, compared to 4 DOFs robot with RCM appropriately registered, to possibly apply undesired forces at the fulcrum.

The present paper deals with the force control of such a comanipulated system. More precisely, the main question under investigation is how to apply a wrench, with a 6DOFs robot, to the handle of an instrument inserted through a fulcrum, in such a way that a user holding the handle feels that a given force is distally applied. This question is specific to assistance to key-hole surgery since, in open surgery, there is no interaction with a fulcrum that may affect the forces applied to the instrument and thus the surgeon's felt forces. Meanwhile, a secondary question concerns the minimization of the forces applied to the fulcrum during such an operation.

The paper is organized as follows. Two strategies for computing the distal wrench are proposed in Sec. II. They are then compared through experiments. Section III describes the experimental methods (setup, virtual fixtures, protocol and indicators). Section IV provides the results, that are further discussed in Sec. V.

\section{TWO CONTROL STRATEGIES}

\section{A. Problem formulation}

Our aim in this paper is to evaluate how to generate distal virtual fixtures for a minimally invasive instrument comanipulated at its proximal end.

As depicted in Fig. 2, the instrument is modeled as a straight line joining the proximal (handle) end $U$ to the distal end $D$ (tip). A frame $\mathscr{F}_{I}=\left(U, \vec{x}_{I}, \vec{y}_{I}, \vec{z}_{I}\right)$, with $\vec{z}_{I}=(1 /\|\overrightarrow{D U}\|) \overrightarrow{D U}$ is 
attached to the instrument. Three wrenches are applied to the instrument during a comanipulated experiment.

Firstly, the user applies a wrench denoted:

$$
\left\{\mathscr{W}_{u}\right\}=\left\{\begin{array}{l}
\vec{F}_{u} \\
\vec{M}_{u}
\end{array}\right\}_{U},
$$

where $\vec{F}_{u}$ is a vector force and $\vec{M}_{u}$ is a vector moment applied at proximal point $U$.

Secondly, the wrench applied by the patient to the instrument through the fulcrum $F$ is noted:

$$
\left\{\mathscr{W}_{f}\right\}=\left\{\begin{array}{l}
\vec{F}_{f} \\
\vec{M}_{f}
\end{array}\right\}_{F},
$$

where $\vec{F}_{f}$ is a vector force and $\vec{M}_{f}$ is a vector moment applied at the insertion point $F$.

Notice that if a 4 DOFs joint model is assumed for the fulcrum, leaving free only three rotations around a fixed point $F$ and one translation along $\vec{z}_{I}$, then the twist representing the velocity of the instrument with respect to a fixed frame, writes, at Point $F$ :

$$
\left\{\mathscr{T}_{I}\right\}=\left\{\begin{array}{c}
\vec{\omega}=\omega_{x} \vec{x}_{I}+\omega_{y} \vec{y}_{I}+\omega_{z} \vec{z}_{I} \\
v_{z} \vec{z}_{I}
\end{array}\right\}_{F} .
$$

Reciprocally, if friction is neglected, then the joint does not dissipate any mechanical power for all the possible velocities of the instrument. This allows to establish [27] that the wrench transmissible through the fulcrum writes:

$$
\left\{\mathscr{W}_{f}\right\}=\left\{\begin{array}{c}
\vec{F}_{f}=F_{f x} \vec{x}_{I}+F_{f y} \vec{y}_{I} \\
\vec{M}_{f}=\overrightarrow{0}
\end{array}\right\}_{F} .
$$

The third wrench applied to the instrument is produced by the comanipulator. In this paper, the robot is supposed to be able of applying a controlled wrench:

$$
\left\{\mathscr{W}_{r}\right\}=\left\{\begin{array}{l}
\vec{F}_{r} \\
\vec{M}_{r}
\end{array}\right\}_{R},
$$

where $\vec{F}_{r}$ is a vector force and $\vec{M}_{r}$ is a vector moment applied at a given proximal point $R$.

The robot shall be programmed to apply virtual fixtures. The virtual fixture generator, by itself, does not fit in the scope of the paper. A conventional approach is supposed to be used: considering the position and orientation of the instrument, the virtual fixture generator shall produce a virtual force that can be either repulsive (forbidden region) or attractive (guide), see examples in Sec. III. In a conventionnal configuration, this vector force $\vec{F}_{v}$ should be applied at a distal point $V$, inside the patient, whose location is also provided by the virtual fixture generator. However, in the configuration on minimally invasive surgery, the robot shall apply a wrench $\left\{\mathscr{W}_{r}\right\}$ at a proximal point $W$ in such a way that the user feels that the virtual wrench $\left\{\mathscr{W}_{v}\right\}$ :

$$
\left\{\mathscr{W}_{v}\right\}=\left\{\begin{array}{c}
\vec{F}_{v} \\
\overrightarrow{0}
\end{array}\right\}_{V}
$$

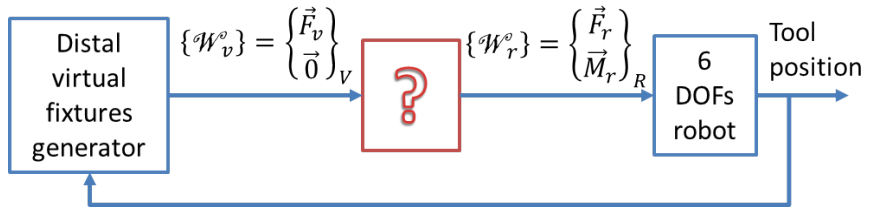

Figure 3. The problem under consideration in this paper: how to compute a proximal wrench $\left\{\mathscr{W}_{r}\right\}$ to be applied by the robot that will be felt by the user as if a wrench $\left\{\mathscr{W}_{v}\right\}$ was distally applied

has been distally applied.

How to compute $\left\{\mathscr{W}_{r}\right\}$ from $\left\{\mathscr{W}_{v}\right\}$ is the central question of this paper, as depicted in Fig. 3. It is important to notice that, from a mechanical point of view, the answer to this question is not unique due to the presence of internal forces. In other words, given a solution $\left\{\mathscr{W}_{r 1}\right\}$ that provides a satisfactory behavior for the robot control, any other solution $\left\{\mathscr{W}_{r 2}\right\}$ that writes:

$$
\left\{\mathscr{W}_{r 2}\right\}=\left\{\mathscr{W}_{r 1}\right\}+\left\{\mathscr{W}_{f 1}\right\}
$$

where $\left\{\mathscr{W}_{f 1}\right\}$ corresponds to a wrench verifying Eq. (4) can result in the same user (felt) wrench. Indeed, the difference between $\left\{\mathscr{W}_{r 2}\right\}$ and $\left\{\mathscr{W}_{r 1}\right\}$ can be totally compensated by the fulcrum wrench without changing the user wrench.

Combining Eq. (7) with Eq. (4), the condition for two robot wrench solutions to be equivalent writes:

$$
\exists \phi_{x} \in \mathbb{R}, \exists \phi_{y} \in \mathbb{R} /\left\{\mathscr{W}_{r 2}\right\}-\left\{\mathscr{W}_{r 1}\right\}=\left\{\begin{array}{c}
\phi_{x} \vec{x}_{I}+\phi_{y} \vec{y}_{I} \\
\overrightarrow{0}
\end{array}\right\}_{F} .
$$

This formulation raises a question on the forces $\phi_{x}$ and $\phi_{y}$ exerted at the fulcrum. Indeed, in the context of minimally invasive surgery, it is desired that these forces are minimized. However, because $\left\{\mathscr{W}_{u}\right\}$ is unknown, and may also include components that are transmissible through the fulcrum (thus affecting $\left.\left\{\mathscr{W}_{f}\right\}\right)$, it is impossible to a priori compute the solution for $\left\{\mathscr{W}_{r}\right\}$ that will minimize $\left\{\mathscr{W}_{f}\right\}$.

In the next, among the infinite number of possible solutions described by Eq. (8), we select two remarkable solutions. The first one, called exact wrench computation, consists in computing $\left\{\mathscr{W}_{r}\right\}$ equal to $\left\{\mathscr{W}_{v}\right\}$. Namely, with this approach, the robot applies at point $R$ the wrench (a force and a moment) that a pure virtual force $\vec{F}_{v}$ exerted at point $V$ would apply at point $R$. The second solution consists in applying a pure force at point $R$ (no moment) with the robot. This solution, which refers to a force-lever model, is chosen mainly because it simplifies the robot design. Indeed, a robot with only 3 actuated joints serially mounted with a passive spherical wrist centered at point $R$ can implement this strategy (see details in Sec. II-D).

\section{B. Exact Wrench Computation (EWC)}

The most immediate way to emulate $\left\{\mathscr{W}_{v}\right\}$ with the robot is to apply with the robot a wrench $\left\{\mathscr{W}_{r}\right\}$ that is equal to the wrench $\left\{\mathscr{W}_{v}\right\}$. This corresponds to the conventional wrench 
moment displacement formula:

$$
\left\{\mathscr{W}_{r}\right\}=\left\{\begin{array}{c}
\vec{F}_{v} \\
\overrightarrow{0}
\end{array}\right\}_{V}=\left\{\begin{array}{c}
\vec{F}_{v} \\
\overrightarrow{R V} \times \vec{F}_{v}
\end{array}\right\}_{R}=\left\{\begin{array}{c}
\vec{F}_{v} \\
-l_{V} \vec{z}_{I} \times \vec{F}_{v}
\end{array}\right\}_{R}
$$

where $l_{V}>0$ is defined by $l_{V} \vec{z}_{I}=\overrightarrow{V R}$.

\section{Lever Model Computation (LMC)}

Another possible approach consists in using the lever principle, i.e. balancing the distal pure force $\vec{F}_{v}$ with a proximal pure force $\vec{F}_{r}$. With this approach, the robot is controlled to apply a wrench with a null moment at point $R\left(\vec{M}_{r}=\overrightarrow{0}\right)$. It shall balance the pure force $\vec{F}_{v}$ applied at point $V$, given the particular kinematic constraint imposed by the fulcrum.

One shall compute $\left\{\mathscr{W}_{r}\right\}$ (with a null moment at point $R$ ) that is mechanically equivalent to $\left\{\mathscr{W}_{v}\right\}$ for all the movements permitted by the fulcrum. In other words, assuming that the kinematic constraint is depicted by Eq. (3), $\left\{\mathscr{W}_{r}\right\}$ shall develop the same mechanical power as $\left\{\mathscr{W}_{v}\right\}, \forall\left\{\omega_{x}, \omega_{y}, \omega_{z}, v_{z}\right\} \in \mathbb{R}^{4}$.

Denoting:

$$
\left\{\mathscr{W}_{r}\right\}=\left\{\begin{array}{c}
F_{r x} \vec{x}_{I}+F_{r y} \vec{y}_{I}+F_{r z} \vec{z}_{I} \\
\overrightarrow{0}
\end{array}\right\}_{R},
$$

it is straightforward to show that the mechanical power developed by $\left\{\mathscr{W}_{r}\right\}$ is:

$$
\begin{aligned}
P_{r} & =\left\{\mathscr{T}_{I}\right\} \odot\left\{\mathscr{W}_{r}\right\} \\
& =\left\{\begin{array}{c}
\omega_{x} \vec{x}_{I}+\omega_{y} \vec{y}_{I}+\omega_{z} \vec{z}_{I} \\
v_{z} \vec{z}_{I}+\overrightarrow{R F} \times \vec{\omega}
\end{array}\right\}_{R} \odot\left\{\begin{array}{c}
F_{r x} \vec{x}_{I}+F_{r y} \vec{y}_{I}+F_{r z} \vec{z}_{I} \\
\overrightarrow{0}
\end{array}\right\}_{R} \\
& =l_{F} \omega_{y} F_{r x}-l_{F} \omega_{x} F_{r y}+v_{z} F_{r z}
\end{aligned}
$$

where $\odot$ stands for the screw scalar product and $l_{V}>l_{F}>0$ is defined by: $l_{F} \vec{z}_{I}=\overrightarrow{F R}$. Similarly, denoting:

$$
\left\{\mathscr{W}_{v}\right\}=\left\{\begin{array}{c}
F_{v x} \vec{x}_{I}+F_{v y} \vec{y}_{I}+F_{v z} \vec{z}_{I} \\
\overrightarrow{0}
\end{array}\right\}_{V},
$$

the mechanical power developed by $\left\{\mathscr{W}_{v}\right\}$ writes:

$$
\begin{aligned}
P_{v} & =\left\{\mathscr{T}_{I}\right\} \odot\left\{\mathscr{W}_{v}\right\} \\
& =-\left(l_{V}-l_{F}\right) \omega_{y} F_{v x}+\left(l_{V}-l_{F}\right) \omega_{x} F_{v y}+v_{z} F_{v z}
\end{aligned}
$$

The wrenches $\left\{\mathscr{W}_{r}\right\}$ and $\left\{\mathscr{W}_{v}\right\}$ are equivalent iff:

$$
\forall \omega_{x}, \omega_{y}, \omega_{z}, v_{z} \quad P_{r}=P_{v}
$$

which is equivalent to:

$$
\left\{\begin{array}{l}
F_{r z}=F_{v z} \\
F_{r x}=-\frac{l_{V}-l_{F}}{l_{F}} F_{v x} \\
F_{r y}=-\frac{l_{V}-l_{F}}{l_{F}} F_{v y}
\end{array}\right.
$$

Thus, denoting the lever factor:

$$
\alpha=\frac{l_{V}-l_{F}}{l_{F}}>0,
$$

the wrench to be applied by the robot for a given force $\vec{F}_{v}$ virtually applied at point $V$ writes:

$$
\left\{\mathscr{W}_{r}\right\}=\left\{\begin{array}{c}
\vec{F}_{r}=-\alpha F_{v x} \vec{x}_{I}-\alpha F_{v y} \vec{y}_{I}+F_{v z} \vec{z}_{I} \\
\vec{M}_{r}=\overrightarrow{0}
\end{array}\right\}_{R} .
$$

\section{Differences between the EWC and LMC approaches}

Two ways of computing $\left\{\mathscr{W}_{r}\right\}$ from a given $\vec{F}_{v}$ virtually applied at point $V$ are given by Eqs. (9) and (17). In theory, they are mechanically equivalent providing that the kinematic constraint imposed by the patient entry point to the instrument verifies Eq. (3). Indeed, it is straightforward to verify that their difference satisfies the necessary condition given by Eq. (8). From a perceptual point of view, the user may not feel any difference although the values of the force/moment components drastically differ: force components along $\vec{x}_{I}$ and $\vec{y}_{I}$ have opposite signs in both Equations while the moment components in Eq. (9) are zeroed in Eq. (17).

In practice, the two computation strategies significantly differ by several aspects. In the next, we list them and explain how they were taken into account to design an experimental set-up allowing for practically investigating these open questions.

i) A first aspect concerns the forces applied by the instrument at the fulcrum. As mentioned earlier, it is impossible to a priori determine which strategy will minimize the forces applied at the fulcrum due to the possible contribution of the unknown user wrench to $\left\{\mathscr{W}_{f}\right\}$. To evaluate this question, an experimental apparatus allowing to independently measuring forces and moments at the fulcrum will be used in Sec. III.

ii) The second aspect that distinguishes the two possible approaches for simulating a distal force $\vec{F}_{v}$ is that, for the LMC approach, it is assumed that deformations induced by tissue elasticity are null, while the EWC approach does not rely on any particular model for the patient-instrument relative kinematics. In practice though, deformations do occur. This could lead to a lack of precision when applying LMC while EWC is still valid. Again, theoretically predicting the effect of this approximation on the quality of force perception for the user is difficult. Rather, an experimental approach will be used in Sec. III to study this question using an apparatus that includes deformations at the fulcrum.

iii) The third difference between the two strategies concerns the data required to compute the robot wrench. For EWC, only the geometry of the robot is to be known, as well as the desired location of $V$ long the instrument axis. Meanwhile, for LMC, $\alpha$ has to be computed from Eq. (16) which, in practice, requires to know where point $F$ stands. In other words, a registration between the robot and the patient is required, which reduces the interest of using a 6-DOFs robot rather than a 4 DOFs RCM robot. In practice, if we want to avoid registration, an arbitrarily constant value $\hat{\alpha}$ can be used instead of $\alpha$, corresponding to an average lever ratio, typically 1 . This strategy will be experimentally evaluated in Sec. III.

iv) The fourth factor that distinguishes the two strategies has a significant impact in practice. While EWC strategy requires 6 actuated DOFs, only 3 actuated DOFs can be sufficient for LMC. Indeed, consider a 6-DOF robot consisting in 3 actuated DOFs serially mounted with a passive (unactuated) 3 DOFs wrist realizing a ball joint at point $R$. With such a robot, the three first actuators are used to produce a desired 


\begin{tabular}{||l|l||}
\hline Workspace & cube $45 \mathrm{~cm}$ in size \\
\hline Maximum force & $31 \mathrm{~N}(8.5 \mathrm{~N}$ continuous $)$ \\
\hline Maximum rotation torque & $3.1 \mathrm{Nm}(1 \mathrm{Nm}$ continuous $)$ \\
\hline Resolution in position & $0.02 \mathrm{~mm}$ \\
\hline Friction & $0.4 \mathrm{~N}$ and $0.07 \mathrm{Nm}$ \\
\hline Inertia & $0.7 \mathrm{~kg}$ and $0.003 \mathrm{~kg} . \mathrm{m}^{2}$ \\
\hline \multicolumn{2}{|c||}{ Table I }
\end{tabular}

Main ViRTuOse 6D SPECIFICATIONS. FRICTION AND INERTIA HAVE BEEN ESTIMATED IN AN AVERAGE POSITION, AT POINT $R$. THEY SHALL BE UNDERSTOOD AS ORDERS OF MAGNITUDE.

$\vec{F}_{r}$ while the passive spherical wrist ensures that $\vec{M}_{r}=\overrightarrow{0}$. Clearly, in the perspective of transferring the technology to a clinical application, being able of exploiting a robot with only three actuators instead of six brings a significant advantage in terms of robot complexity, weight, inertia and cost. For this reason, such a particular robot kinematic configuration, with 3 actuators only, will be used to implement LMC in the next section.

\section{EXPERIMENTAL METHODS}

Experiments have been conducted in order to compare the efficiency of the two proposed approaches.

\section{A. Experimental setup overview}

The comanipulator used for the experiments is a 6 active DOFs haptic device which main specifications are listed Table I (model: Virtuose 6D - provider: Haption [28]). Its kinematics comprises 3 first pivot joints that position a point $R$ and a 3 joint wrist realizing a ball joint around $R$. Depending on the strategy to be experimented, the wrist joints will be actuated or not. Thanks to a fine mechanical design involving low friction cable transmissions, low inertia fiber carbon links, and gravity compensation springs, the Virtuose $6 \mathrm{D}$ robot allows to control $\left\{\mathscr{W}_{r}\right\}$ in open loop with a high precision.

A rod with a handle is connected to the robot last body in order to emulate a surgical tool. To emulate the patient, a dedicated apparatus has been designed and fabricated. The rod is connected to an intermediate mechanical part through a cylindrical joint. This intermediate part is connected to a fixed drum through four springs as it can be seen on Fig. 4 . The springs stiffness has been experimentally tuned in such a way that the obtained behavior is similar to those of an incision point or a natural orifice of a patient. Namely, it is not a perfect link, as the fulcrum point $F$ can be displaced to simulate tissue deformations, while friction appears through the cylindrical link. In order to be able of measuring $\left\{\mathscr{W}_{f}\right\}$, the drum is mounted on a force sensor.

As we want to assess the efficiency of the virtual fixtures for the two proposed control laws, the space that is behind the drum is hidden by a screen. Therefore, the users are not able to directly visualize the tool tip position. A global view of the setup is given in Fig. 5.

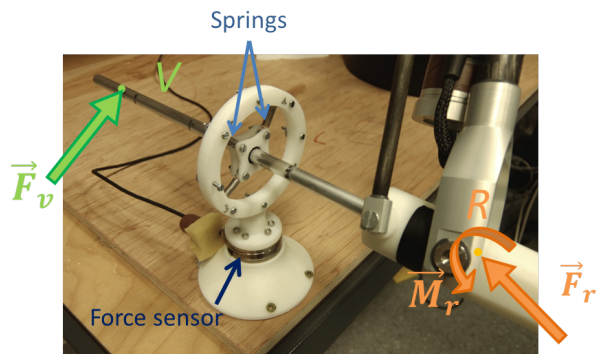

Figure 4. Close up on the mechanical device that emulates the anatomical constraint.

\section{B. Virtual Fixtures used for the experiments}

In order to cover a large range of surgical scenarios, where virtual fixtures correspond to either repulsive regions in the aim of increasing safety (e.g. [10]) or attractive paths to guide an instrument (e.g. [7]), three types of virtual fixtures have been implemented for the experiments.

- Repulsive spherical region

A virtual spherical object, centered at a fixed point $C$, with a radius $r$ is designed. The projection $C^{\prime}$ of $C$ on the instrument main axis, namely the line $(D U)$, is first computed. Then, Point $V$ where the force should be applied in case of intersection between the instrument and the sphere is set to:

$$
V= \begin{cases}D & \text { if }\left\|\overrightarrow{U C^{\prime}}\right\|>\|\overrightarrow{C D}\| \\ C^{\prime} & \text { otherwise }\end{cases}
$$

When $V$ belongs to the sphere, then an intersection is detected and a force is applied. The force is radial and proportional to the radial depth of $V$ into the sphere, which writes:

$$
\vec{F}_{v}= \begin{cases}k_{s} \frac{(r-\|\overrightarrow{C V}\|)}{\|\overrightarrow{C V}\|} \overrightarrow{C V} & \text { if } r \geq\|\overrightarrow{C V}\|>0 \\ \overrightarrow{0} & \text { otherwise }\end{cases}
$$

where $k_{s}$ is the stiffness of the sphere. This is illustrated in Fig. 6.

\section{- Repulsive plane}

A virtual plane $\Pi$ is defined, thanks to a point $P \in \Pi$ and a normal vector $\vec{n}$, pointing from the forbidden region to the

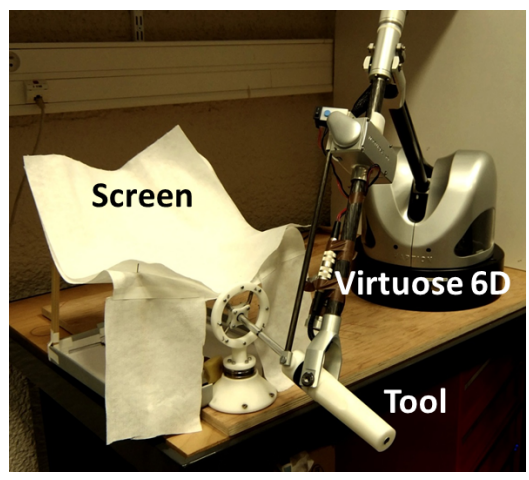

Figure 5. Complete experimental set-up. 


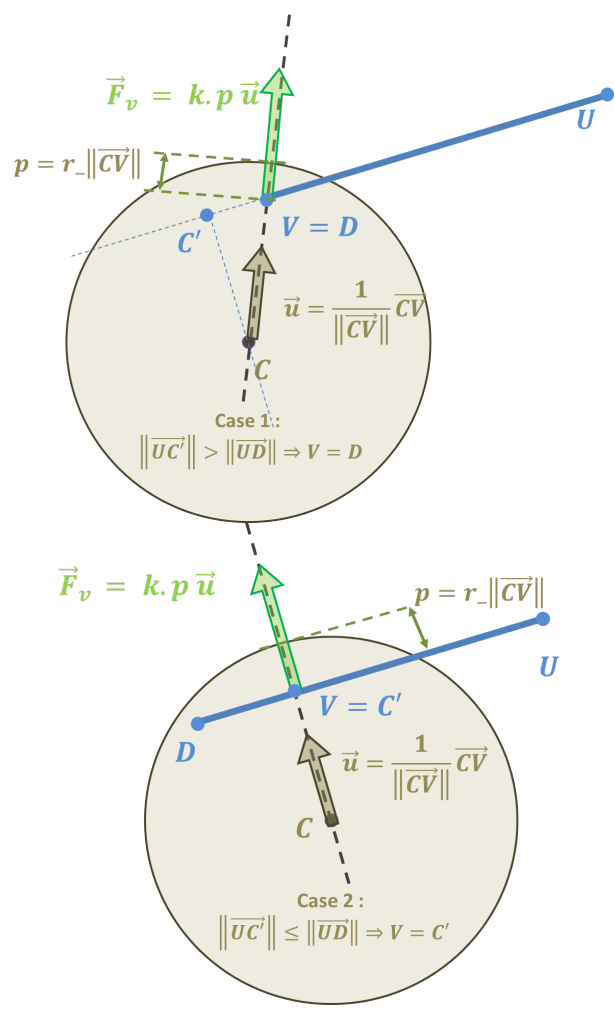

Figure 6. Computation of the virtual force $\vec{F}_{v}$ to simulate the interaction between the instrument and a virtual sphere

authorized region. The virtual plane applies no forces when the instrument distal tip $D$ is the authorized region and a repulsive force when $D$ is in the forbidden region. Point $V$ is set to the instrument tip $D$. Its projection on $\Pi$, denoted $V^{\prime}$, is first computed. The force is normal to the plane and proportional to the penetration of $V$ beyond $\Pi$, which writes:

$$
\vec{F}_{v}= \begin{cases}k_{p} \overrightarrow{V^{\prime} V} & \text { if } \overrightarrow{V V^{\prime}} \cdot \vec{n}>0 \\ \overrightarrow{0} & \text { otherwise }\end{cases}
$$

where $k_{p}$ is the stiffness of the plane.

- Attractive line

A virtual line $(\Delta)$ is defined thanks to a point $P \in(\Delta)$ and a unit direction vector $\vec{u}$. The role of the virtual fixture is to help the user keeping the instrument distal tip $D$ on $(\Delta)$. To this aim, Point $V$ is set to the instrument tip $D$. Its projection on $(\Delta)$, denoted $V^{\prime}$, is first computed. The force then simply writes:

$$
\vec{F}_{v}=k_{l} \overrightarrow{V V^{\prime}}
$$

where $k_{l}$ is a stiffness.

\section{Experimental protocol}

Fourteen naive subjects have been enrolled in the study, aged 13 to 60 . The subjects were not paid for the experiment. None of them have declared suffering from a sensorimotor deficiency. They had to perform 3 different exercises. Firstly, a Ball Sorting Out (BSO) exercise during which 4 virtual spheres with different sizes were simulated by the robot

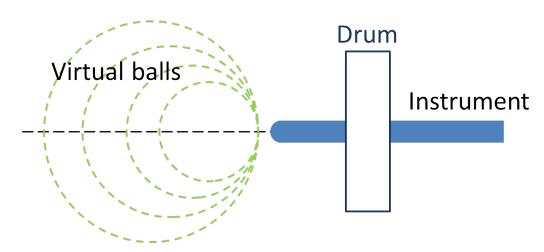

Figure 7. Position of the different virtual balls with respect to the drum.

while the subjects were asked to blindly palpate them and to sort them out from the smallest one to the largest one. These balls have the same stiffness, that was tuned to provide an easy contact detection while experimentally preserving stability $\left(k_{s}=200 \mathrm{~N} / \mathrm{m}\right)$. The ball radius are log-distributed: $R=\{1.5,2.4,3.75,6\} \mathrm{cm}$. There is only one virtual ball at a time in the workspace. The balls are directly facing the entry point. Whatever the ball size, its most proximal point is always located at a given place, as illustrated in Fig. 7. Therefore, in order to evaluate the ball size, the subjects must palpate laterally. The balls are presented to the subject in a random order, then the subjects can switch to a previously presented ball, as many times as they want. Once they think they have sorted out the balls, they stop the exercise and name the ball from the smallest to the largest.

Secondly, a Plane Sorting out (PSO) exercise during which 4 planes with identical geometry but different stiffnesses were simulated by the robot while the subjects were asked to blindly palpate them and to sort them out from the softest one to the hardest one. The plane stiffness values were tuned to respect stability constraint and to range from a "soft feeling" to a "hard feeling". They are log-distributed: $k_{p}=\{200,340,580,1000\} N / m$. The virtual plane presents no geometrical particularity with the other elements of the experiments. Note that theoretically, palpating only one point of the plane could be enough for a subject to infer the stiffness. However, all the subjects decided to palpate the plane at several locations and to push against the plane. The PSO exercise unfolds as the BSO exercise. It stops when the subjects name the plane from the softest to the stiffest.

Thirdly, a Line Following (LF) exercise during which the subjects were asked to draw a straight line with the instrument tip "following the robot indication". In this exercise, an initial configuration is given to the tool by the operator. The subject must then make the tip of the tool following a straight line at his/her own pace, back and forth (3 times). The line direction is unknown, and the subject can use only the force feedback to follow the minimum force path. The line stiffness was tuned to $k_{l}=300 \mathrm{~N} / \mathrm{m}$ so as to respect experimental stability conditions.

For the three exercises, the following general instructions are given to the subjects: the general context of the study (key hole surgery) is explained to the subjects, with particular emphasis on the prostate biopsy procedure. This is aimed at making them aware that during the exercises, forces applied at the fulcrum should be minimized. They are explicitly asked to pay attention to this objective during all the exercises. The subjects do not know anything more about the goal of the 

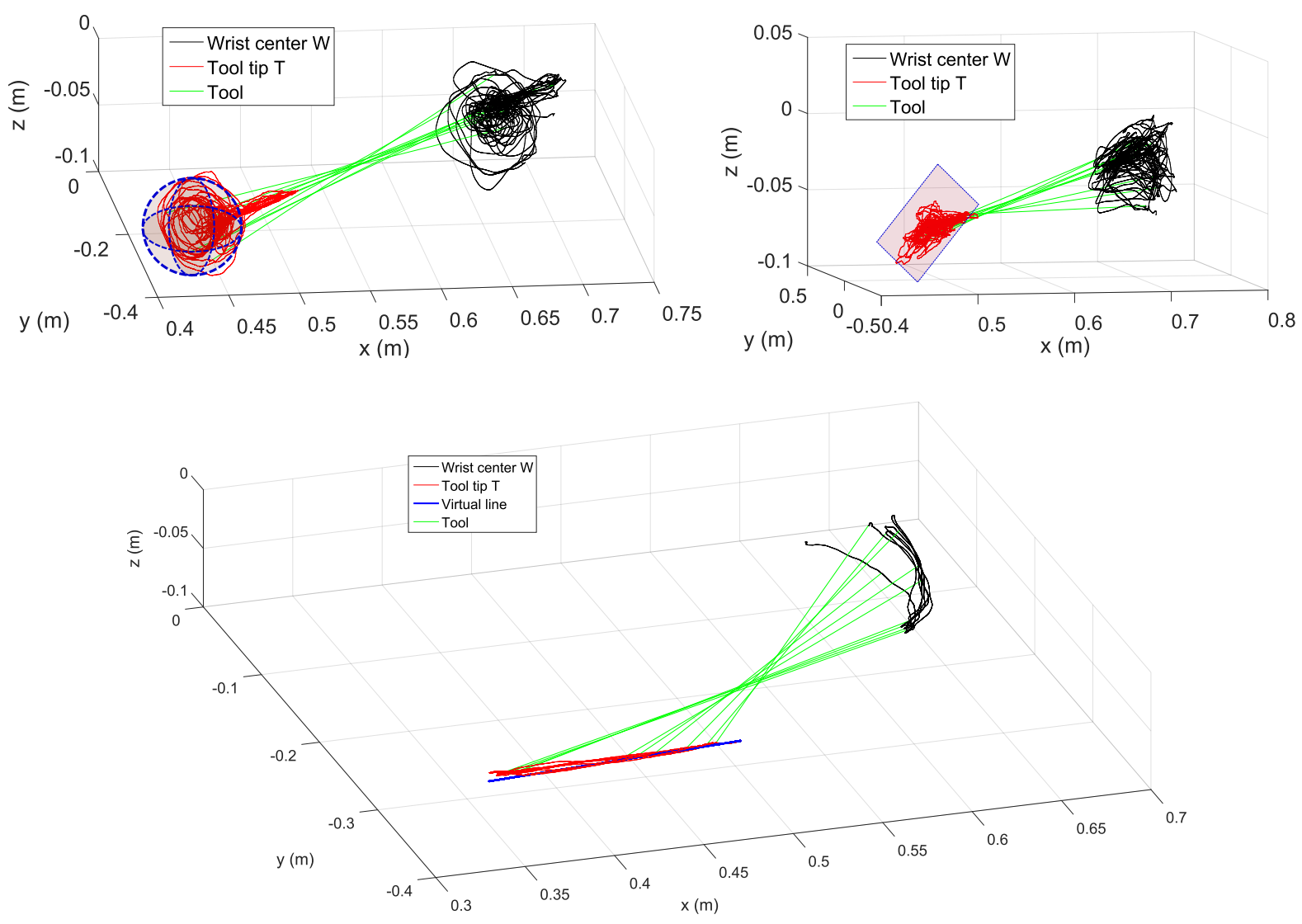

Figure 8. Trajectories observed for different points belonging to the tool during the BSO exercise (upper left), the PSO exercise (upper right) and the line following exercise (bottom). Results are shown for one randomly selected subject for illustration purpose.

experience. They are asked to do each exercise twice: one trial for each controller, chosen in a random order for the three exercise, thus avoiding an influence of the learning effect on the statistical results. However, the subjects are not aware that two different controllers are being used. They are simply asked to repeat twice each exercise. Notice also that the subjects do not see the instrument tip, which is hidden behind the screen. They see only the proximal part of the instrument, the insertion apparatus and the robot. This way their perception of virtual objects is only obtained through the kinesthetic feedback.

\section{Indicators}

In order to assess the differences between EWC and LMC, several physical variables are recorded during the experiments. Among them, those that are representative of the gesture quality (forces at the fulcrum, positioning precision, movements smoothness) and the perception quality (duration and adequacy of the sorting out exercises) are used as performance indicators. Namely, the selected indicators are:

- for all the exercises:

- the task completion time $t_{\text {total }}$, which is the time needed by the subject to perform the exercise.

- the Spectral Arc Length (SAL) of the trajectories of point $R$ as defined in [29]. SAL is the opposite of the length along the spectral curve of a movement. Not only it is an image of the complexity of the movement Fourier magnitude spectrum, but it is also dimensionless and independent of the movement magnitude and duration. Its value is negative; the closer it is to zero, the simpler the movement Fourier spectrum is and thus the smoother the movement is.

- the maximal force $F_{\max }$ applied by the tool on the fulcrum.

- the mean force $F_{\text {mean }}$ applied by the tool on the fulcrum.

- for the BSO and PSO exercises only, a score is computed to quantify the adequacy of the answers. Namely, when the subject sorts out the spheres or the planes without any errors, a score $\sigma=2$ is given; when all the spheres or planes are correctly sorted out except for one inversion between two consecutive items, a score $\sigma=1$ is given; when there are more errors than one single inversion, a score $\sigma=0$ is given.

- for LF exercise:

- the largest distance $d_{\max }$ from the tool tip $D$ to the virtual line.

- the mean distance $d_{\text {mean }}$ from the tool tip $D$ to the virtual line. 


\begin{tabular}{||l||c|c||c|c||}
\hline \multicolumn{1}{|c||}{} & \multicolumn{2}{c||}{ BSO } & \multicolumn{2}{c||}{ PSO } \\
& EWC & LMC & EWC & LMC \\
\hline \hline Correct answers & 7 & 9 & 6 & 10 \\
\hline Answers with one inversion & 1 & 2 & 3 & 2 \\
\hline Answers with more inversions & 4 & 1 & 3 & 0 \\
\hline Mean score $\sigma$ & 1.25 & 1.67 & 1.25 & 1.83 \\
\hline Std. dev. for $\sigma$ & 0.96 & 0.65 & 0.87 & 0.39 \\
\hline p-value (Wilcoxon) & \multicolumn{2}{|c||}{0.19} & \multicolumn{4}{c||}{0.053} \\
\hline
\end{tabular}

Table II

ACCURACY OF THE SUBJECTS FOR THE SORTING OUT EXERCISES

\section{EXPERIMENTAL RESUlts}

Figure 8 gives, for illustration purposes, example trajectories of points $T$ and $R$ during each of the three exercises.

In order to evaluate the influence of the control method on the performance indicators, statistical tests are used to determine whether a difference experimentally observed between two groups of measured values is statistically significant or not. As usually admitted in the human motion analysis literature, tests resulting in a p-value smaller than 0.05 is considered to be statistically significant. Note that due to some technical issues, the recorded sensory data was corrupted for two subjects during the BSO and PSO exercises and for one subject during the LF exercise. Therefore, only twelve (resp. thirteen) data sets have been included for the statistical analysis of the BSO and PSO (resp. LF) exercises.

Table II analyzes the accuracy of the subjects' answers for the sorting out exercises. For both exercises, the measured average score is higher with LMC than with EWC. However, the observed difference between the score means is not statistically significant $(p>0.05)$. Not that the score $\sigma$ being non-continuous, a Wilcoxon signed rank test has been used to compute the p-values.

Figure 9 shows the indicators for trajectory smoothness, fulcrum force (mean and max) and experiment duration averaged across subjects, for the three exercises. For all the four indicators, a slight difference between LMC and EWC can be observed, in either way. However, results seem globally similar. This similarity is confirmed in Table III. In this table, p-values computed from paired t-tests are used to compare EWC and LMC. All the slight differences observed have no statistical significance since none of the p-values is below 0.05 .

For the Line Following exercise, the results for the precision (maximum error $d_{\max }$ and mean error $d_{\text {mean }}$ ) are given in Fig. 10. Here, it can be observed that EWC clearly outperforms LMC. This is confirmed through the statistical test made

\begin{tabular}{||l||c|c|c||}
\hline & BSO & PSO & LF \\
\hline \hline$t_{\text {total }}$ & 0.055 & 0.234 & 0.591 \\
\hline SAL & 0.150 & 0.845 & 0.883 \\
\hline$F_{\text {max }}$ & 0.245 & 0.530 & 0.946 \\
\hline$F_{\text {mean }}$ & 0.408 & 0.055 & 0.564 \\
\hline \hline
\end{tabular}

Table III

RESULTS OF THE STUDENT PAIRED-T-TEST PERFORMED TO COMPARE THE EWC AND LMC PERFORMANCE FOR THE FOUR INDICATORS USED IN FIG. 9 AND THE THREE EXERCISES. between EWC and LMC since p-values equal 0.0004 for $d_{\max }$ and 0.0001 for $d_{\text {mean }}$.

Finally, subjects were asked, at the end of the session, whether they had felt a difference between the two repetitions of each exercise. The answers fell in three categories:

- one subject felt more comfortable with LMC but wasn't able to explain precisely what was different from the EWC command;

- two subjects felt that EWC provided "more help" than LMC, only for the LF exercise. However, they were not able to differentiate both commands in BSO and PSO exercises;

- ten subjects didn't notice any difference between two repetitions of any exercise.

This indicates that, from the user point of view, there is not much difference between the two controllers.

\section{DisCusSION}

Most indicator comparisons between EWC and LMC exhibit no statistically significant difference. From a mathematical point of view, these results shall not be interpreted as a proof for both controllers to perform equally. Rather, these results indicate that the average difference of performance between the two controllers, if any, is too small to be observed through these experiments, given the inter-subject variability. In other words, there is a high probability that the actual difference for these indicators is, indeed, small. This is confirmed
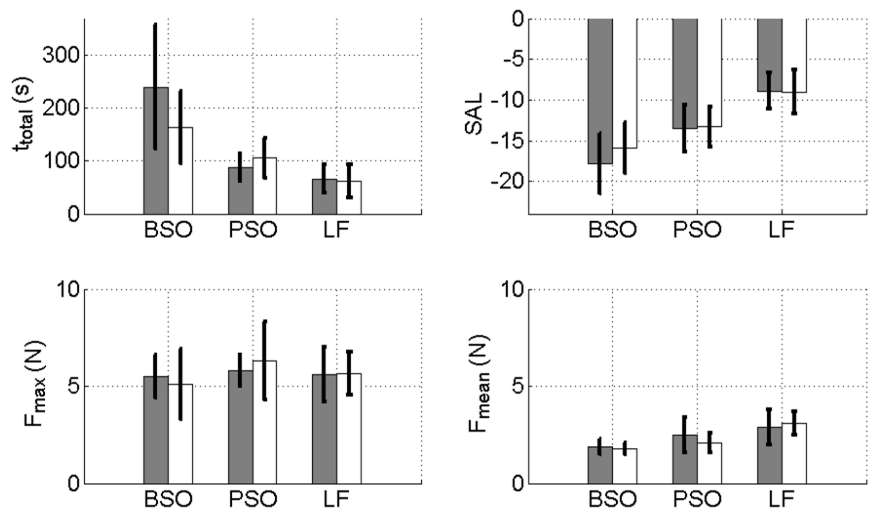

Figure 9. Average value of the different indicators across the subjects, for the three exercises (gray bars: LMC; white bars: EWC). Black lines represent the standard deviation.

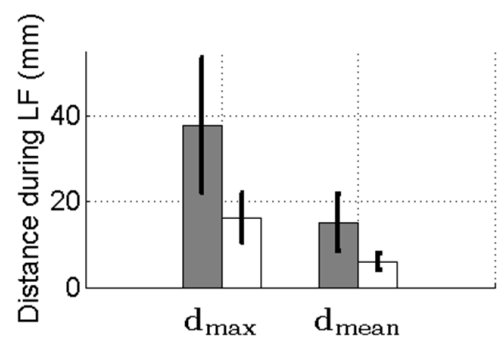

Figure 10. Average value of the precision indicators across the subjects, for the LF exercise (gray bars: LMC; white bars: EWC). Black lines represent the standard deviation. 
by the fact that most users did not notice a difference between the two controllers.

It is worth noticing that the forces applied at the fulcrum are, in average, rather low for all the sorting out exercises, under both conditions. Meanwhile, much larger forces were exerted on the virtual objects while large movements were produced by the users, see typical examples in Fig. 8. This result was surprising to the authors as, from Eq. (8), large differences in fulcrum forces were expected. We interpret the relative smallness of fulcrum forces as resulting from the fact that subjects were explicitly asked to avoid exerting large forces at the fulcrum. They were trying to precisely maintain point $F$ still. A typical motor behavior for precisely controlling the position of a point under disturbances is to increase the impedance at this point along the direction of the disturbance force. In these experiments, disturbance forces to be rejected to lie in the $\left(\vec{x}_{i}, \vec{y}_{i}\right)$ plane. It may thus be hypothesized that the subjects selected a motor behavior leading to a high impedance at point $F$ in the $\left(\vec{x}_{i}, \vec{y}_{i}\right)$ plane. If the subjects' impedance was high at point $F$, then this would also explain why they essentially did not feel any difference between the two controllers, while actually applying wrenches to balance the fulcrum-compatible robot wrenches.

As for the LF exercise, the precision of the tip trajectory control is higher with EWC than with LMC, with statistical significance, see Fig. 10. For this exercise, subjects' attention is brought to the tip, that shall precisely follow a straight line. Therefore, it can be hypothesized that subjects tend to increase the impedance at the instrument tip. This may be conflicting with the high impedance requirement at point $F$. As a result, it can be observed that the fulcrum forces are larger, in average, during LF exercise than during sorting out exercises (see Fig. 9). Note that when following a line, the distal virtual force should be minimized by the subject, while when palpating, eventually large virtual forces should be applied to distinguish between stiffness and sizes. In that sense, the fact that the fulcrum forces are larger for the LF exercise is not a scale effect due to higher distal virtual forces, but rather a consequence of a poorer control at the fulcrum level.

The hypothesis that the fulcrum forces control is degraded during the LF exercise may explain why EWC performs better than LMC. Indeed, remind that EWC control is the exact solution of the problem, independently from any entry point model. therefore, whatever the fulcrum displacement, EWC provides a feedback that exactly corresponds to a pure distal force. Rather, LMC is based on two hypotheses: first, the impedance at $F$ shall be high (infinite) and second, the coefficient $\alpha$ shall be close to 1 . These conditions are poorly verified during the $L F$ experiments, which may explain the difference between the two modes in terms of tip precision, which depends on how well a subject can interpret the emulated distal force.

Further investigation is certainly needed to evaluate the precision performance for tip guidance. First, remind that the experiments are here performed blindly, while the subject doesn't know in advance the trajectory to follow. In a real surgical configuration, not only the instrument tip is controlled under visual guidance, but also the geometrical constraint is a priori known by the user. Performing LF experiments under these more realistic conditions would probably tend to lower the difference of performance between the two controllers, since the visual feedback would equally contribute to an increased precision in both conditions.

\section{CONCLUSION}

Comanipulation and virtual fixtures offer a demonstrated interest for assistance to surgery. Meanwhile, little attention has been paid so far to the emulation of distal force from proximal comanipulation.

In this paper, this question is demonstrated to admit an infinite number of solutions, due to the fulcrum constraint. Experimental results comparing two selected approaches do not lead to a clear difference between them. From a practical point of view, this similarity may advantageously lead to select LMC rather than EWC. Indeed, contrarily to EWC, LMC can be implemented with a three actuators arm combined to a passive spherical wrist. An example of such a device is given in [9].

As the paper concerns the generation of a proximal force to emulate a distal force, the experiments were designed in such a way that the subjects focus on the haptic feedback produced by the robot. For this reason, no visual feedback was used. This is a limitation in the perspective of exploiting the results for assistance to surgery, where both a visual feedback and a haptic feedback will be simultaneously available. A further investigation is to be performed considering now the combination of visual feedback and force feedback. A base for this future work can be found in [30], where in comparison to unimodal tests, the combination of both information is shown to lead to increased performances, in the context of surgery.

\section{REFERENCES}

[1] J. J. Abbott, P. Marayong, and A. M. Okamura, "Haptic virtual fixtures for robot-assisted manipulation," in Robotics research, pp. 49-64, Springer Berlin Heidelberg, 2007.

[2] J. Abbott and A. Okamura, "Virtual fixture architectures for telemanipulation," in IEEE International Conference on Robotics and Automation (ICRA), vol. 2, pp. 2798-2805 vol.2, Sept 2003.

[3] C. Poquet, P. Mozer, G. Morel, and M.-A. Vitrani, "A novel comanipulation device for assisting needle placement in ultrasound guided prostate biopsies," in IEEE/RSJ International Conference on Intelligent Robots and Systems (IROS), pp. 4084-4091, Nov 2013.

[4] C. Moore, M. Peshkin, and J. Colgate, "Cobot implementation of virtual paths and 3d virtual surfaces," IEEE Transactions on Robotics and Automation, vol. 19, pp. 347-351, Apr 2003.

[5] S. Bowyer, B. Davies, and F. Rodriguez y Baena, "Active constraints/virtual fixtures: A survey," IEEE Transactions on Robotics, vol. 30, pp. 138-157, Feb 2014.

[6] S. J. Morel, G. and M.-A. Vitrani, "Comanipulation," in Medical Robotics (J. Troccaz, ed.), no. ISBN: 978-1-84821-33, Wiley, 2012.

[7] O. Schneider and J. Troccaz, "A six degree of freedom passive arm with dynamic constraints (padyc) for cardiac surgery application: Preliminary experiments," Computer-Aided Surgery, special issue on medical robotics, vol. 6, pp. 340-351, 2001.

[8] M. Li, A. Kapoor, and R. Taylor, "A constrained optimization approach to virtual fixtures," in IEEE/RSJ International Conference on Intelligent Robots and Systems (IROS), pp. 1408-1413, Aug 2005. 
[9] C. Poquet, P. Mozer, M.-A. Vitrani, and G. Morel, "An endorectal ultrasound probe comanipulator with hybrid actuation combining brakes and motors," IEEE/ASME Transactions on Mechatronics, vol. 20, pp. 186196, Feb 2015.

[10] M. Jakopec, F. Rodriguez y Baena, S. Harris, P. Gomes, J. Cobb, and B. L. Davies, "The hands-on orthopaedic robot acrobot: Early clinical trials of total knee replacement surgery," IEEE Transactions on Robotics and Automation, vol. 19, pp. 902-911, Oct 2003.

[11] B. Davies, F. Rodriguez y Baena, A. Barrett, M. Gomes, S. Harris, M. Jakopec, and J. P. Cobb, "Robotic control in knee joint replacement surgery," Proceedings of the institution of mechanical engineers part H-Journoal of engineering in medicine, vol. 221, pp. 71-80, Jan 2007.

[12] J. Cobb, J. Henckel, K. Brust, y. B. F. Rodriguez, S. Harris, M. Jakopec, A. Barrett, and B. Davies, "Accuracy provides enduring functional improvement in arthroplasty:18 months results of robotic assisted unicompartmental knee replacement," in 7th International Annual Meeting of Computer Assisted Orthopaedic Surgery (CAOS), pp. 183-185, 2007.

[13] D. Nawabi, M. Conditt, A. Ranawat, N. Dunbar, J. Jones, S. Banks, and S. Padgett, "Haptically guided robotic technology in total hip arthroplasty: a cadaveric investigation," Proceedings of the Institution of Mechanical Engineers. Part H, Journal of engineering in medicine, vol. 227, no. 3, pp. 302-9, 2013 Mar.

[14] V. Francoise, A. Sahbani, and G. Morel, "A comanipulation device for orthopedic surgery that generates geometrical constraints with real-time registration on moving bones," in IEEE International Conference on Robotics and Biomimetics (ROBIO), pp. 38-43, Dec 2011.

[15] F. Huang, F. Mussa-Ivaldi, C. Pugh, and J. Patton, "Learning kinematic constraints in laparoscopic surgery," IEEE Transactions on Haptics, vol. 5, pp. 356-364, Fourth 2012.

[16] G. Guthart and J. Salisbury, "The intuitive telesurgery system : overview and application," in International Conference on Robotics and Automation (ICRA), pp. 618-621, april 2000.

[17] R. Konietschke, U. Hagn, M. Nickl, S. Jorg, A. Tobergte, G. Passig, U. Seibold, L. Le-Tien, B. Kubler, M. Groger, F. Frohlich, C. Rink, A. Albu-Schaffer, M. Grebenstein, T. Ortmaier, and G. Hirzinger, "The dlr mirosurge - a robotic system for surgery," in IEEE International Conference on Robotics and Automation (ICRA), pp. 1589-1590, May 2009.

[18] T. Ortmaier and G. Hirzinger, "Cartesian control issues for minimally invasive robot surgery," in Proceedings. 2000 IEEE/RSJ International Conference on Intelligent Robots and Systems (IROS), vol. 1, pp. 565571 vol.1, 2000.

[19] L. Michelin, E. Dombre, and P. Poignet, "Geometrical control approaches for minimally invasive surgery," in Medical Robotics, Navigation and Visualization (MRNV'04), (Remagen, Allemagne), pp. 152159, march 2004.

[20] M. Vitrani, H. Mitterhofer, G. Morel, and N. Bonnet, "Robust ultrasound-based visual servoing for beating heart intracardiac surgery," in ICRA'07 - IEEE International Conference on Robotics and Automation, (Rome, Italie), pp. 3021-3027, 2007.

[21] H. Azimian, R. Patel, and M. Naish, "On constrained manipulation in robotics-assisted minimally invasive surgery," in $3 r d$ IEEE RAS and EMBS International Conference on Biomedical Robotics and Biomechatronics (BioRob), pp. 650-655, Sept 2010.

[22] C. D. Pham, F. Coutinho, A. C. Leite, F. Lizarralde, P. J. From, and R. Johansson, "Analysis of a moving remote center of motion for robotics-assisted minimally invasive surgery," in 2015 IEEE/RSJ International Conference on Intelligent Robots and Systems (IROS), pp. 1440-1446, Sept 2015.

[23] A. Krupa, G. Morel, and M. de Mathelin, "Achieving high precision laparoscopic manipulation through adaptive force control.," Advanced Robotics, vol. 18, no. 9, pp. 905-926, 2004.

[24] S. Low and L. Phee, "A review of master-slave robotic systems for surgery.," in MICCAI-Medical Image Computing and Computer-Assisted Intervention, vol. 2, pp. 145-152, Springer, september 2004.

[25] N. Zemiti, T. Ortmaier, M. Vitrani, and G. Morel, "A force controlled laparoscopic surgical robot without distal force sensing," in 9th International Symposium on Experimental Robotics (ISER), (Singapore), June 2004.

[26] N. Zemiti, G. Morel, A. Micaelli, B. Cagneau, and D. Bellot, "On the force control of kinematically defective manipulators interacting with an unknown environment," IEEE Transactions on Control Systems Technology, vol. 18, pp. 307-322, March 2010.
[27] J. Angeles, Fundamentals of Robotics Mechanical Systems. Springer, 2014.

[28] Haption, "http://www.haption.com/."

[29] S. Balasubramanian, A. Melendez-Calderon, and E. Burdet, "A robust and sensitive metric for quantifying movement smoothness," IEEE Transactions on Biomedical Engineering, vol. 59, pp. 2126-2136, Aug 2012.

[30] V. Francoise, A. Sahbani, A. Roby-Brami, and G. Morel, "Assistance to bone milling: A tool mounted visual display improves the efficiency of robotic guidance," in 35th Annual International Conference of the IEEE Engineering in Medicine and Biology Society (EMBC), pp. 6252-6256, July 2013. 